\title{
Oral findings in Midline Syndrome: A case report and literature review
}

\author{
Victòria Tallón-Walton ${ }^{1}$, Pekka Nieminen ${ }^{2,3}$, Sirpa Arte ${ }^{2,3}$, Josep $\mathbf{M}^{\mathrm{a}}$ Ustrell-Torrent $^{4}$, Patricia Carvalho- \\ Lobato ${ }^{1}, M^{\text {a }}$ Cristina Manzanares-Céspedes ${ }^{1}$
}

${ }^{1} \mathrm{DDS}$ PhD, MD PhD Human Anatomy and Embryology Unit, Campus de Bellvitge, Barcelona University, Spain

${ }^{2} \mathrm{MD}$ PhD Institute of Dentistry, Biomedicum, University of Helsinki, Finland

${ }^{3}$ MD PhD Department of Oral and Maxillofacial Diseases, Helsinki University Central Hospital, Finland

${ }^{4}$ MD PhD Odonto-Stomatology Department, Faculty of Dentistry, Barcelona University, Spain

Correspondence:

Human Anatomy and Embryology Unit

Campus de Bellvitge,

Barcelona University 5305,

Pavelló de Govern,

$5^{a}$ planta. Feixa Llarga, $s / n$

08907 -L'Hospitalet del Llobregat.

Barcelona-Spain

mcmanzanares@ub.edu

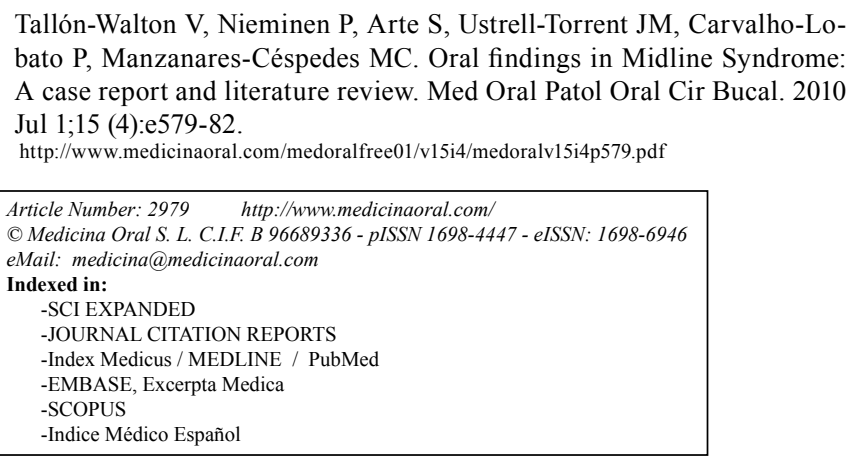

\begin{abstract}
We describe a female patient with a midline syndrome. The patient presents agenesis of the corpus callosum, encephalocele, iris coloboma, hypertelorism, submucosal cleft palate and dental anomalies. Despite being very characteristic, her phenotypical traits do not coincide exactly with those reported to date in the literature. The karyotype and the molecular cytogenetic study do not show mutations. We identify the presence of dental anomalies in the mother and other family members, not being identified MSX1 and PAX9 mutations that could the related with their etiology. Despite the fact that dental agenesis has been related to a large number of other malformation syndromes and congenital conditions, dental anomalies have only rarely been mentioned when reporting midline syndromes. These dental phenotypical traits, present in the patient and her family, could be considered part of the midline syndrome in carriers as well as in the patients.
\end{abstract}

Key words: Craniofacial development, dental anomalies, hypodontia, craniofacial anomalies.

\section{Introduction}

Embryological development is under a strict system of control involving hundreds of known genes. Mutations of these genes, as well as environmental factors, have been associated with the etiology of certain congenital syndromes and conditions. Specifically, some have been related to human midline and bilateral symmetry defects (1-3), for instance the Opitz G/BBB syndrome, hypospadias-dysphagia syndrome, a rare condition associated with midline abnormalities such as laryngotracheo-esophageal cleft, cleft lip and palate, heart anomalies, genito-urinary defects and agenesis of the corpus callosum (4). Its genetic etiology is complex, consisting of both X-linked recessive and autosomal dominant forms (5).

The oro-facial-digital syndrome is a heterogeneous group 
of conditions. At least thirteen different types have been described, based on characteristic clinical manifestations. Common features are the presence of oral, facial and digital alterations, as well as an X-linked inheritance pattern and mild manifestations in carrier females (6-8). Holoprosencephalia is a rare condition characterized by the presence of a series of congenital cerebral anomalies as well as alterations of the facial structures; specifically, it is due to the failure of the differentiation of the prosencephalon. The severity of the condition ranges from minor signs to forms incompatible with human life. Children affected present a characteristic facial morphology, comprising microcephalia and hydrocephalia, hypotelorism, flattened nose with one nostril, short nose with a pronounced saddle-shape, cleft lip and/or palate and agenesis of the incisors or a solitary central incisor. The etiology has been related to both genetic and teratogenic factors (9). This group of congenital conditions also includes midline facial defects with hypertelorism, a group of rare, heterogenic craniofacial disorders characterized by the presence of a bifid nose (10).

We present a case of midline syndrome showing phenotypical characteristics that do not coincide entirely with those described to date in the literature.

\section{Clinical Description}

An eight-year-old female patient with a midline syndrome is described in the present report. The karyotype and the molecular cytogenetic study using telomeric markers of all the chromosomes, performed at the CIAC (Genetic laboratory of the ECEMC (http://iier.is.ciii.es) (3), with a resolution of 550-850 bands, did not show noticeable anomalies (46,XX.ish multiprobe-T).

The patient presented an alteration in the spheno-ethmoidal region (see the lateral teleradiography of the cranium (Fig. 1c)), hypertelorism, iris coloboma, agenesis of the corpus callosum and encephalocele. No other development anomalies have been observed. The first and second toes on both feet were unusually far apart. In the oral cavity, she presented a partial central cleft lip and an alteration of the insertion of the orbicularis oris muscle of the lips which was rectified surgically during the first year of life, and a submucosal cleft palate. In the primary dentition, the fusion of the maxillary left lateral incisor and a supernumerary peg-shaped tooth were identified (Fig. 1a). Large-scale dental crowding was also seen, mainly in the anterior upper maxillary region (Fig. 1d). The panoramic radiography showed agenesis of the maxillary second bicuspids, as well as

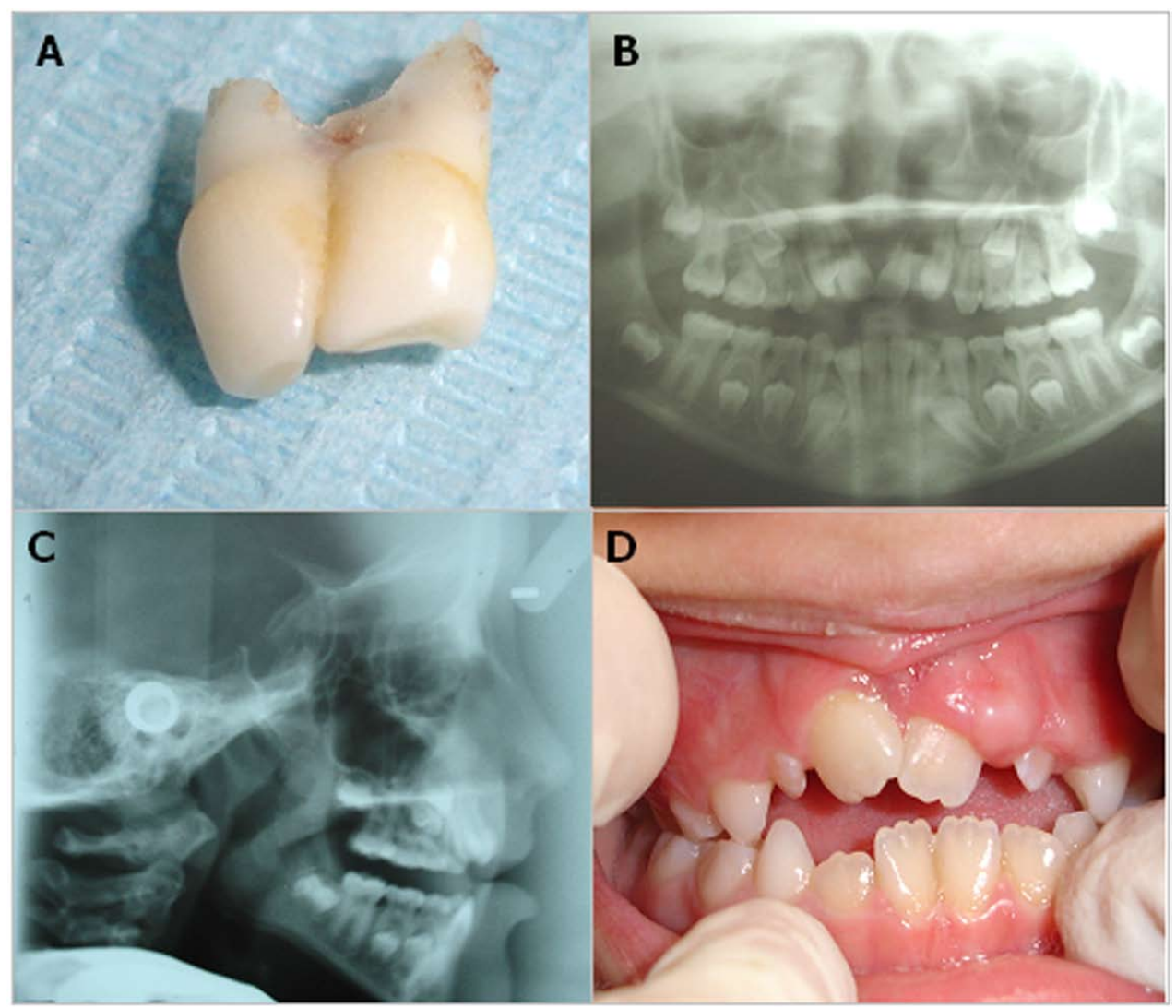

Fig. 1. a: Temporary maxillar lateral incisive fused with a supernumerary peg-shaped tooth. b: Panoramic radiography of the patient in which the agenesis of the maxillary right second premolars and the peg-shaped maxillar left lateral incisor can be observed. c: Lateral teleradiography of the patient where the absence of the Sella turcica can be observed. d: Front photograpy of the patient where an important crowding at incisive sector is observed. 
a possible microdontia of the mandibular ones, which had not yet erupted. The most notable shape anomaly was the presence of a peg-shaped maxillary left lateral incisor (Fig. 1b). No structural anomalies were observed in either primary or permanent dental organs.

The patient's family history revealed agenesis of the mandibular left second premolar in the mother and the absence of the mandibular right second premolar in her sister. In these family members we didn't find any mutations in the coding regions or in the exon-intron junctions of PAX9 and MSX1 genes, that could be related with the etiology of tooth agenesis and other dental anomalies. The existence of dental anomalies among the remaining members of the family is unknown, due to the absence of complementary diagnostic information or because of prosthetic rehabilitation, which renders its assessment impossible. Two first-degree female cousins also present the same characteristic separation of the first and second toes.

The mother had also suffered two miscarriages. The first one was reported as "spontaneous", with no data available on the condition of the male foetus or its phenotypical characteristics. The second abortion was therapeutic, due to the miscarriage of a male foetus presenting an omphalocele. The karyotype and the molecular cytogenetic study did not present obvious anomalies.

\section{Discussion}

The syndrome presented by the patient is similar to the ones described as midline syndrome, but does not express all the phenotypical characteristics described in the literature or in the reference databases $(2,3)$. This group of syndromes, characterized by the presence of alterations at midline and bilateral level, includes amongst others, the oro-facial-digital syndrome (a heterogeneous group of genetic disorders that have in common the presence of oral, facial and digital alterations) $(2,3,7)$, and the Opitz syndrome (a genetic disorder with a wide range of clinical expressions, the main signs being hypotelorism, hypospadias, wide nasal root, and dysphonic crying; the presence of cleft lip or palate has also been described, and thoracic and abdominal alterations as well as genito-urinary anomalies) $(7,11)$. A recent clinical study of thirty-one individuals with midline facial defects and hypertelorism found a strong association between anomalies of the skull and facial bones, related to a disturbance in the development of the frontonasal process, ear and larynx, and the central nervous system. No dental anomalies were reported in these patients (10).

The frontonasal prominence is composed of the mesenchyme, derived from the cranial neural crest, surrounded by epithelia derived from the neuroectoderm of the forebrain and facial ectoderm. The neuroectoderm influences the patterning of the neural crest inside the craniofacial bones; the blocking of the molecular signals issued from the neuroectoderm leads to holoprosencephaly and other craniofacial malformation syndromes (12).

Among the extra-oral traits of the midline syndromes, the most notable in our case is an alteration in the spheno-ethmoidal region. The endochondral ossification of the cranial base starts around the 12th week of development, at the basal portion of the occipital bone, followed by the body of the sphenoid bone around the 16th week, and then by the ethmoid bone. In this way the complete ossification of the sella turcica is complete by birth. The ossification advances, both in the endochondral and the endomembranous bones, leaving open growth areas between the craniofacial bones: the mandibular symphysis and the metopic suture remain open until twenty-four months after birth (1). The mid-palatine suture ensures the mediolateral growth of the maxillaries $(1,4,12)$. The spheno-occipital synchondrosis in the middle of the clivus closes and disappears at puberty. Here we established a correlation with the patient's craniofacial anomalies, since these are the cranial structures that showed the most obvious alterations.

The agenesis of the corpus callosum is related to a defect in the closure of the neural tube (12). This entity is associated with other malformations of the central nervous system such as holoprosencephaly, encephalocele, meningocele and the Dandy-Walker condition. To a great extent, these malformations are due to the absence of differentiation of the neuroblasts and the cerebral cortex, which occurs after the closure of the neural tube and which, in a similar way, is related to the formation of the corpus callosum. These disorders emerge between the tenth and the 20th week of pregnancy, at the time when the cranial base bones start their ossification. The nasal encephalocele may appear at the moment of birth in the form of hypertelorism and widening of the nasal bones (13). Therefore, a certain temporal co-incidence is observed in the genesis of the spheno-ethmoidal defect and the corpus callosum agenesis identified in this patient. Moreover, Tapadia et al. (12) described a wide range of craniofacial anomalies which are associated with the forebrain malformation in the holoprosencephaly syndromes related to mutations in the SHH gene, despite the fact that no clear genotypephenotype correlation has been found. Neural crest cell populations send and receive complex signals, especially from the superficial ectoderm, the neuroepithelium and the pharyngeal endoderm, though a variety of signalling pathways (Shh, Wnt, Fgf and Bmp) are also involved in the odontogenesis (14).

The presence of dental anomalies has only rarely been mentioned in midline syndromes. In a recent clinical study by Gil-da-Silva-Lopes et al. (10) of thirty-one individuals with midline facial defects, no reference was 
made to congenital dental anomalies. However, dental agenesis has been related to a large number of other malformation syndromes and congenital conditions. Specifically, the submucosal cleft palate has been associated with dental agenesis, the most frequently affected dental organs being the mandibular second premolars, followed by the maxillary lateral incisors and the second premolars. Ectopic eruption and transpositions; peg-shaped maxillary lateral incisors and supernumerary teeth have also been reported (15). Our patient and her family presented some of these anomalies.

As regards the genetic etiology of the syndrome described here, since the mother was the carrier, we suggest that the presence of dental agenesis, as well as some characteristic facial features also found in the patient's mother and sister, could be considered as a part of the phenotypical manifestations of this condition. Up to now PAX9 and MSX1 are ones of main candidate genes for dental agenesis in humans $(16,17)$; not being identified mutations in the coding regions or in the exonintron junctions of these genes in the mother that could be related with congenital missing teeth.

Omphalocele is a congenital defect of the midline of the abdominal wall which causes the extrusion of the abdominal content. It may be present as an isolated trait, in association with anomalies in other organs - such as hypospadias- and systems, or as a phenotypical characteristic of certain syndromes, such as the BeckwithWiedemann syndrome (2).

The analysis of the phenotypes of congenital syndromes or conditions is an important step in the assessment of the function of related genes. This analysis is of great interest in the study of closely related genes (for example, those that belong to the same protein complex or signalling pathway), since mutations of these genes are related to similar phenotypical patterns (18). This kind of study may help to broaden our understanding of the etiology of certain syndromes and conditions which are unknown at present, such as the one described here.

\section{References}

1. López-Gracia ML, Ros MA. Left-right asymmetry in vertebrate development. Adv Anat Embryol Cell Biol. 2007;188:1-121.

2. Online Mendelian Inheritance in Man [Internet]. Baltimore: Johns Hopkins University; [updated June 2009; cited October 2009]. Available from: http://www.ncbi.nlm.nih.gov/omim/ .

3. Instituto de Investigación de Enfermedades Raras [Internet]. Madrid: Instituto de Salud Carlos III; [updated June 2009; cited October 2009]. Available from: http://iier.is.ciii.es.

4. Sedano HO, Gorlin RJ. Opitz oculo-genital-laryngeal syndrome (Opitz BBB/G compound syndrome). Am J Med Genet. 1988;30:847-9, 851

5. Parashar SY, Anderson PJ, Cox TC, McLean N, David DJ. Multidisciplinary management of Opitz G BBB syndrome. Ann Plast Surg. 2005;55:402-7.

6. Edwards M, Mulcahy D, Turner G. X-linked recessive inheritance of an orofaciodigital syndrome with partial expression in females and survival of affected males. Clin Genet. 1988;34:325-32.
7. Gurrieri F, Franco B, Toriello H, Neri G. Oral-facial-digital syndromes: review and diagnostic guidelines. Am J Med Genet A. 2007;143A:3314-23.

8 . Toriello HV. Heterogeneity and variability in the oral-facial-digital syndromes. Am J Med Genet Suppl. 1988;4:149-59.

9. Dubourg C, Bendavid C, Pasquier L, Henry C, Odent S, David V. Holoprosencephaly. Orphanet J Rare Dis. 2007;2:8.

10. Gil-da-Silva-Lopes VL, Maciel-Guerra AT. A clinical study of 31 individuals with midline facial defects with hypertelorism and a guideline for follow-up. Arq Neuropsiquiatr. 2007;65:396-401.

11. Brooks JK, Leonard CO, Coccaro PJ Jr. Opitz (BBB/G) syndrome: oral manifestations. Am J Med Genet. 1992;43:595-601.

12. Tapadia MD, Cordero DR, Helms JA. It's all in your head: new insights into craniofacial development and deformation. J Anat. 2005;207:461-77.

13. Paller AS, Pensler JM, Tomita T. Nasal midline masses in infants and children. Dermoids, encephaloceles, and gliomas. Arch Dermatol. 1991;127:362-6.

14. Gene expression in tooth [Internet]. Helsinki: Tooth and Craniofacial Development, Group of the Development Biology Programme, Institute of Biotechnology. University of Helsinki; [updated June 2009; cited October 2009]. Available from: http:// www.bite-it.helsinki.fi .

15. Helióvaara A, Ranta R, Rautio J. Dental abnormalities in permanent dentition in children with submucous cleft palate. Acta Odontol Scand. 2004;62:129-31.

16. Vastardis H, Karimbux N, Guthua SW, Seidman JG, Seidman CE. A human MSX1 homeodomain missense mutation causes selective tooth agenesis. Nat Genet. 1996;13:417-21.

17. Stockton DW, Das P, Goldenberg M, D'Souza RN, Patel PI. Mutation of PAX9 is associated with oligodontia. Nat Genet. 2000;24:18-9.

18. Oti M, Brunner HG. The modular nature of genetic diseases. Clin Genet. 2007;71:1-11.

\section{Acknowledgements}

The authors would like to thank the members of the family for their participation. The study was supported by the COST action B-23 ORAL FACIAL DEVELOPMENT AND REGENERATION, and by an ACESBELL-06 grant awarded by the Research Committee of the Bellvitge Campus of Health Sciences, University of Barcelona. 